Document downloaded from:

http://hdl.handle.net/10251/152254

This paper must be cited as:

Salas-Molina, F.; Rodriguez-Aguilar, JA.; Pla Santamaría, D. (2019). Characterizing compromise solutions for investors with uncertain risk preferences. Operational Research. 19(3):661-677. https://doi.org/10.1007/s12351-017-0309-6

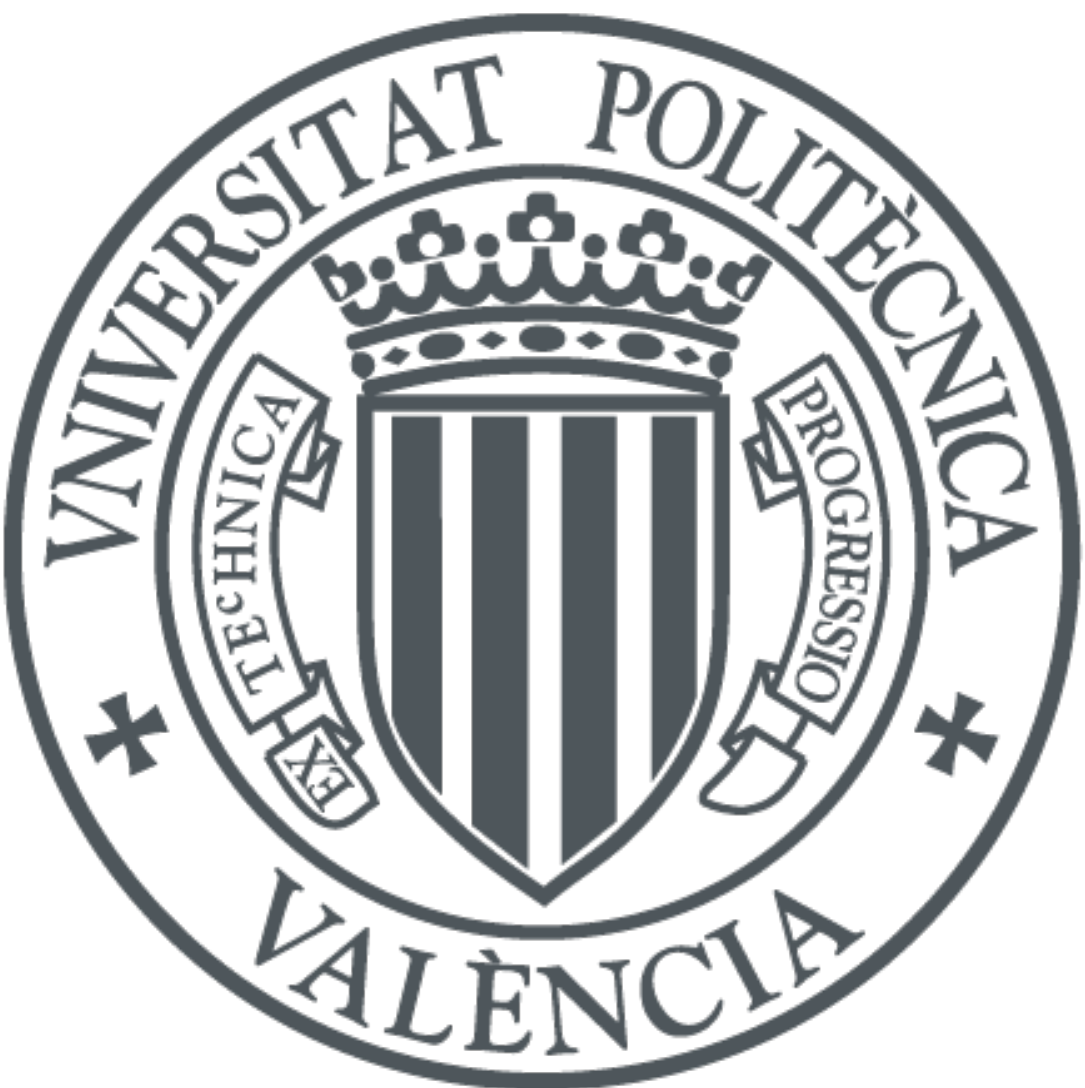

The final publication is available at

https://doi.org/10.1007/s12351-017-0309-6

Copyright Springer-Verlag

Additional Information 


\title{
Characterizing compromise solutions for investors with uncertain risk preferences
}

\author{
Francisco Salas-Molina . \\ Juan A. Rodriguez-Aguilar . \\ David Pla-Santamaria
}

the date of receipt and acceptance should be inserted later

\begin{abstract}
The optimum portfolio selection for an investor with particular preferences was proven to lie on the normalized efficient frontier between two bounds defined by the Ballestero (1998) bounding theorem. A deeper understanding is possible if the decision-maker is provided with visual and quantitative techniques. Here, we derive useful insights as a way to support investor's decision-making through: (i) a new theorem to assess balance of solutions; (ii) a procedure and a new plot to deal with discrete efficient frontiers and uncertain risk preferences; and (iii) two quality metrics useful to predict long-run performance of investors.
\end{abstract}

Keywords: Finance; portfolio selection; compromise programming; discrete efficient-frontiers; performance prediction.

\section{Introduction}

Investment decision makers not only care about profit but also about the amount of risk accepted to achieve that profit. In portfolio selection, profit and risk can be measured by the mean and the variance (or the standard

Francisco Salas-Molina

Hilaturas Ferre, S.A., Les Molines, 2, 03450 Banyeres de Mariola, Alicante, Spain

E-mail: francisco.salas@hifesa.com

Juan A. Rodriguez-Aguilar

IIIA-CSIC, Campus UAB, 08913 Cerdanyola, Catalonia, Spain

E-mail: jar@iiia.csic.es

David Pla-Santamaria

Escuela Politécnica Superior de Alcoy, Ferrándiz y Carbonell, s/n, 03801 Alcoy, Alicante, Spain

E-mail: dplasan@upv.es 
deviation) of portfolio returns. In the search for the best portfolio, Compromise Programming proposed that utility optimization was considered as a distance minimization problem (Zeleny, 1982; Yu, 1985) that has been widely used in many recent works (Bilbao-Terol et al., 2006; Amiri et al., 2011; PlaSantamaria and Bravo, 2013; Ballestero et al., 2015)

However, individual or collective investors may have particular risk preferences different to an average investor, i.e., one unbiased towards profitability or risk. This bias is summarized by Ballestero (1998) through the use of a parameter reflecting the investor's preference. From that, a bounding theorem was proposed by the author to determine the bounds for approximating the optimum portfolio on the efficient frontier for an investor with particular risk preferences. Here, efficient frontier refers to the set of non-dominated portfolios in terms of profitability and risk. In an attempt to reduce the number of available solutions, Ballestero's theorem introduces risk preferences in the optimization problem to provide a bounded solution. However, this theorem does not provide an optimum, but an approximation to the optimum characterized by two points in the efficient frontier delimiting an efficient set of portfolios.

Subsequent works have followed two separated ways. On the one hand, some of them have successfully applied this theorem for selecting portfolios for mutual funds (Ballestero and Pla-Santamaria, 2004) and for eliciting balanced solutions by using an additive linear-quadratic composite metric (Ballestero, 2007; Bravo et al., 2012). On the other hand, a number of related works have recently mentioned Ballestero's theorem as an important contribution to portfolio selection (Ehrgott et al., 2004; Steuer et al., 2007; Xidonas et al., 2012). However, little attention has been paid to the geometry of risk preferences, its relationship with the efficient frontier and its utility to enhance the understanding of the multi-objective decision-making process.

The main goal of this paper is to characterize Ballestero's bounding theorem to enhance its understanding and implications for investment decision makers through:

- a new theorem to assess balance of solutions for different metrics.

- a procedure and a new stairs plot to deal with discrete efficient frontiers and risk preference uncertainty.

- two quality metrics to predict long-run performance of investors.

The structure of this paper is as follows. Section 2 presents the Ballestero's bounding theorem that we characterize using two metrics to obtain the best compromise solutions in Section 3. Next, Section 4 compares linear and quadratic metrics which leads to a new theorem, namely, the balance theorem. An algorithm and a new plot is proposed in Section 6 to obtain solutions for discrete efficient frontiers and uncertain risk preferences. Both the algorithm an the new plot will be illustrated with an example. In Section 7, two quality measures are proposed to predict long-run investor performance. Finally, we elaborate some conclusions in Section 8. 


\section{Background : Ballestero's bounding theorem}

Since Markowitz (1952), portfolio selection is usually approached through mean-variance (E-V) efficient frontier techniques. Each point in this efficient frontier represents an alternative portfolio that produces higher profitability and lower risk than any alternative portfolio in the E-V space. It is said, then, that this portfolio dominates the alternative one. In order to avoid meaningless comparison, Ballestero proposed the efficient frontier to be built through the use of the following indexes: (i) index of profitability, $\theta_{1}$; (ii) index of safety, $\theta_{2}$, which are defined as follows:

$$
\begin{gathered}
\theta_{1}=\frac{E-E_{\min }}{E_{\max }-E_{\min }} \\
\theta_{2}=\frac{V_{\min }-V}{V_{\min }-V_{\max }}
\end{gathered}
$$

where $(E, V)$ is a point of the mean-variance efficient frontier, $\left(E_{\max }, V_{\min }\right)$ is the ideal point and $\left(E_{\min }, V_{\max }\right)$ is the anti-ideal point. Note that the index of safety, $\theta_{2}$, is computed as the reciprocal of the index of risk or, equivalently, index of safety $=1$ - index of risk. These indexes, $\theta_{1}$ and $\theta_{2}$, transform the $\mathrm{E}-\mathrm{V}$ space in a normalized profitability-safety (E-S) space where $0 \leq \theta_{1} \leq 1$ and $0 \leq \theta_{2} \leq 1$.

The efficient frontier takes the form $T\left(\theta_{1}, \theta_{2}\right)=0$ and the investor's utility function is expressed as $U\left(\theta_{1}, \theta_{2}\right)$. Then, if $U_{\theta_{1}}$ and $U_{\theta_{2}}$ are the partial derivatives of $U$ with respect to $\theta_{1}$ and $\theta_{2}$, the marginal rate of substitution (MRS) is defined as:

$$
\operatorname{MRS}\left(\theta_{1}, \theta_{2}\right)=\frac{-d \theta_{2}}{d \theta_{1}}=\frac{U_{\theta_{1}}}{U_{\theta_{2}}}
$$

As argued in Ballestero (1998), a number of common assumptions are usually accepted in economics which are enumerated as follows:

- A1: More is better, in the sense of increasing profitability and safety.

- A2: Differentiability of $U$.

- A3: MRS monotonically decreases as $\theta_{1}$ increases.

- A4: Convexity, regarding the efficient set of portfolios.

- A5: Differentiability of $T$.

- A6: $\theta_{2}$ is a decresing function of $\theta_{1}$ along $T$, that is, $d \theta_{2} / d \theta_{1}<0$.

- A7: Strict concavity, that is, $d^{2} \theta_{2} / d \theta_{1}^{2}<0$.

- A8: The Lagrangean maximum of the investors utility function $U$ is given by the intersection of $T$ with $U_{\theta_{1}} / U_{\theta_{2}}=T_{\theta_{1}} / T_{\theta_{2}}$ where $T_{\theta_{1}}$ and $T_{\theta_{2}}$ are the partial derivatives of $T$ with respect to $\theta_{1}$ and $\theta_{2}$ and it exists on the efficient frontier.

Moreover, it is assumed that, in the investor's utility function, the MRS has a stable and constant value $r_{0}$ along the path $\theta_{1}=\theta_{2}$, which is called the investor's particular risk preference. For instance, an investor willing to lose 1.2 marginal units of safety for increasing her profitability in one marginal 
unit it is said to have $r_{0}=1.2$ and to be a risky investor. On the other hand, an investor willing to lose 0.5 marginal units of safety for increasing her profitability in one marginal unit it is said to have $r_{0}=0.5$ and to be a conservative investor. From the previous set of assumptions and definitions, the Ballestero's bounding theorem is expressed as follows:

Theorem 1 The Langrangean maximum $M$ of the investor's utility $U$ on the efficient frontier $T\left(\theta_{1}, \theta_{2}\right)=0$ with $\theta_{1} \geq 0$ and $\theta_{2} \geq 0$ is bounded between the points $L_{\infty}$ and $L$ defined as:

$-L_{\infty}$ is the intersection of $\theta_{1}=\theta_{2}$ with $T\left(\theta_{1}, \theta_{2}\right)$.

- $L$ is the maximum of the linear utility $V=r_{0} \theta_{1}+\theta_{2}$ subject to $T\left(\theta_{1}, \theta_{2}\right)$.

It is interesting to point out that $L_{\infty}$ does not depend on the particular investor's preferences, expressed here as $r_{0}$, but $L$ does. The reader interested in the proof of this theorem is referred to Ballestero (1998). The underlying idea behind the Ballestero's bounding theorem is approximating solutions (optimum portfolio selections) for an investor with particular risk preferences. In this sense, it generalizes a previous model by Ballestero and Romero (1996), for the case of an average investor without bias for profits or risk. To this end, risk preferences are expressed through risk parameter $r_{0}$, resulting in the transformation of Yu's bounds $(\mathrm{Yu}, 1973)$ into the Ballestero's bounds $L_{\infty}$ and $L$, the latter depending on $r_{0}$.

As a result, the utility of the Ballestero's bounding theorem roots in the possibility to precisely delimit the efficient set of portfolios according to the particular bias for risk of an investor. This theorem has been recently used in portfolio selection research (Ballestero and Pla-Santamaria, 2004; Ballestero, 2007; Bravo et al., 2012). Next we characterize this theorem to the selection of the best portfolio according to particular preferences.

\section{Characterizing Ballestero's bounding theorem for different metrics}

The main goal of this section is to provide a visual and quantitative characterization of Ballestero's bounding theorem for different metrics. This characterization consists of: (i) providing an optimal solution within the bounds $L$ and $L_{\infty}$; and (ii) improving understanding of the selection of best portfolios through graphical tools.

The concept of ideal point is at the core of compromise programming (Zeleny, 1982; Yu, 1985; Ballestero and Romero, 1998). In portfolio selection, the ideal point in the normalized E-S space is $(1,1)$, because the goal is to maximize profit and safety. Since this ideal point is usually unfeasible, it is necessary to look for compromise solutions by minimizing the distance to this ideal point. A general distance function between two bidimensional points $P_{1}=\left(x_{1}, y_{1}\right)$ and $P_{2}=\left(x_{2}, y_{2}\right)$, is the Minkowski distance of order $h$ defined as:

$$
\left(\left|x_{1}-x_{2}\right|^{h}+\left|y_{1}-y_{2}\right|^{h}\right)^{1 / h}
$$


Then, by computing the distance between the ideal point $(1,1)$ and a particular point in the efficient frontier $\left(\theta_{1}, \theta_{2}\right)$, we are in a position to determine whether a given portfolio is better than another. Moreover, when considering particular preferences defined by $r_{0}$, distances must be computed between $\left(r_{0}, 1\right)$, as the new ideal point, and $\left(r_{0} \theta_{1}, \theta_{2}\right)$, as the new particular point in the efficient frontier. From that, a family of normalized distance functions that includes parameter $r_{0}$ determining the investor's risk preferences (Ballestero and Romero, 1998) can be expressed as:

$$
\mathcal{L}_{h}=\left[r_{0}^{h} \cdot\left(1-\theta_{1}\right)^{h}+\left(1-\theta_{2}\right)^{h}\right]^{1 / h}
$$

Note that $\mathcal{L}_{1}$ is the Manhattan distance, $\mathcal{L}_{2}$ is the Euclidean distance, and $\mathcal{L}_{\infty}$ is the Chebyshev distance. They are the most used distances in practice for interpretation and computational reasons (Ringuest, 1992; Ballestero, 2007). In order to characterize Theorem 1, we rely on the concept of isometric curves in the normalized $E-S$ space (Zeleny, 1982; Yu, 1985; Ballestero and Romero, 1998), defined by varying a parameter over the previous family of distance functions to the ideal point. The CP objective function is given by the family of distances defined in (5) to be minimized subject to an efficient frontier, which is equivalent to maximize the following Yu-Zeleny utility (Ballestero, 2007):

$$
U=K-\mathcal{L}_{h}
$$

where $K$ is a constant sufficiently large to assure non-negativity. From that, we are in a position to obtain the best solutions derived from Ballestero's theorem for both Manhattan and Euclidean distances.

\subsection{Characterization of the solution for Manhattan distances}

The Manhattan distance between two points in a bidimensional space is the sum of the absolute values of the differences between the first and second components of each point. Given two points $\left(x_{1}, y_{1}\right)$ and $\left(x_{2}, y_{2}\right)$, their Manhattan distance is calculated as:

$$
\left|x_{1}-x_{2}\right|+\left|y_{1}-y_{2}\right| \text {. }
$$

It can be graphically represented by the strictly horizontal and vertical path between two points located in an imaginary grid. The Manhattan distance is linear and this fact facilitates computations within an optimization problem. In practice, isometric points in terms of Manhattan distances can be represented by lines in a E-S space. Indeed, practitioners can graphically derive the optimal portfolio by plotting the line with slope $r_{0}$ that is tangent to the efficient frontier as the following theorem formally states.

Theorem 2 The Lagrangean maximum $M$ of the investor's utility:

$$
U_{1}=K-\mathcal{L}_{1}=K-r_{0}\left(1-\theta_{1}\right)-\left(1-\theta_{2}\right)
$$


is the solution to $r_{0} \theta_{1}+\theta_{2}-b_{M}=0$, with $b_{M}>0$, being the intercept of the tangent to the efficient frontier $T\left(\theta_{1}, \theta_{2}\right)=0$ between the $L$ and $L_{\infty}$ bounds, with $\theta_{1} \geq 0$ and $\theta_{2} \geq 0$.

Proof A family of parallel isometric lines with slope $r_{0}$, that is, with the same particular preferences, can be obtained by varying a parameter $b$ over:

$$
r_{0} \theta_{1}+\theta_{2}=b .
$$

Hence, there is only a value $b_{M}>0$ such that the line defined by $r_{0}$ and $b_{M}$ is tangent to the efficient frontier $T\left(\theta_{1}, \theta_{2}\right)$ between $L$ and $L_{\infty}$. In this tangential point, $r_{0} \theta_{1}+\theta_{2}-b_{M}=T\left(\theta_{1}, \theta_{2}\right)$ and the condition for the Lagrangean maximum, $U_{\theta_{1}} / U_{\theta_{2}}=T_{\theta_{1}} / T_{\theta_{2}}$, holds. Then, the maximum utility portfolio is given by $r_{0}$ and $b_{M}$ as shown in Figure 1.

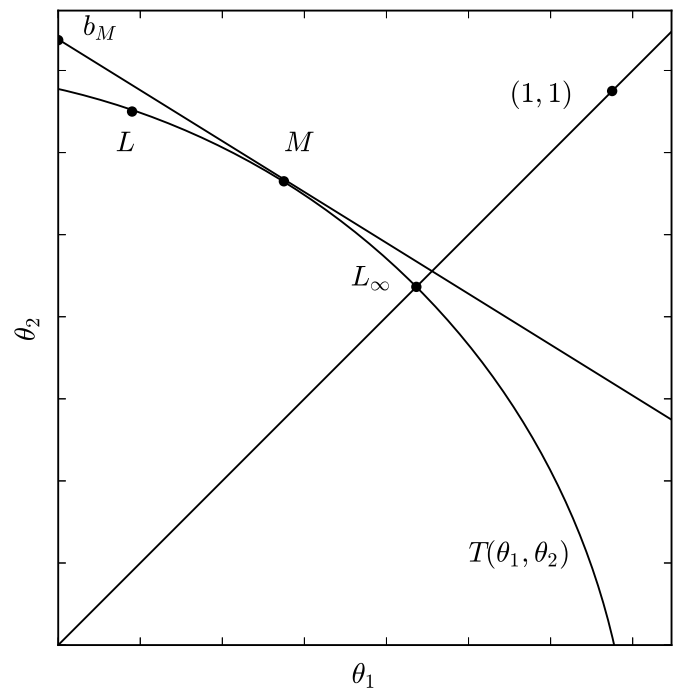

Fig. 1 Normalized efficient frontier $T\left(\theta_{1}, \theta_{2}\right)$ and position of relevant points in the characterization for Manhattan distances

3.2 Characterization of the solution for Euclidean distances

The Euclidean distance between two points in a bidimensional space is the square root of the sum of the squared differences between the first and second components of each point. Given two points $\left(x_{1}, y_{1}\right)$ and $\left(x_{2}, y_{2}\right)$, their 
Euclidean distance is calculated as:

$$
\sqrt{\left(x_{1}-x_{2}\right)^{2}+\left(y_{1}-y_{2}\right)^{2}} \text {. }
$$

It can be graphically represented by the straight line between two points. The Euclidean distance is non-linear and this fact makes computations within an optimization problem more difficult than in the linear case. In practice, isometric points in terms of Euclidean distances can be represented by ellipses in a E-S space. Indeed, practitioners can graphically derive the optimal portfolio by plotting the ellipse with horizontal semiaxis depending on $r_{0}$ that is tangent to the efficient frontier as the following theorem formally states.

Theorem 3 The Lagrangean maximum $M$ of the investor's utility

$$
U_{2}=K-\mathcal{L}_{2}=K-\left[r_{0}^{2}\left(1-\theta_{1}\right)^{2}+\left(1-\theta_{2}\right)^{2}\right]^{1 / 2}
$$

is the solution to $r_{0}^{2}\left(\theta_{1}-1\right)+\left(\theta_{2}-1\right)^{2}-d_{M}^{2}=0$, with $d_{M}>0$, being $d_{M}$ the semiaxis of the ellipse centered in the ideal point $(1,1)$ and tangent to $T\left(\theta_{1}, \theta_{2}\right)$, between $L$ and $L_{\infty}$, with $\theta_{1} \geq 0$ and $\theta_{2} \geq 0$.

Proof A family of isometric ellipses centered in $(1,1)$, with particular preferences defined by $r_{0}$, can be obtained by varying a parameter $d$ over:

$$
r_{0}^{2}\left(\theta_{1}-1\right)^{2}+\left(\theta_{2}-1\right)^{2}=d^{2} .
$$

This family of ellipses is characterized by having the same ratio $a / b$, between the horizontal semiaxis $(a)$ and the vertical semiaxis $(b)$, which depends only on $r_{0}$, since equation (12) can be rewritten as:

$$
\frac{\left(\theta_{1}-1\right)^{2}}{d^{2} / r_{0}^{2}}+\frac{\left(\theta_{2}-1\right)^{2}}{d^{2}}=1
$$

where $a=d / r_{0}$ is the horizontal semiaxis and $b=d$ is the vertical semiaxis. Then, $a / b=1 / r_{0}$ or $b / a=r_{0}$.

Hence, there is only a value $d_{M}>0$ such that the isometric ellipse is tangent to the efficient frontier $T\left(\theta_{1}, \theta_{2}\right)$. In the tangential point, given by $r_{0}^{2}\left(\theta_{1}-1\right)^{2}+\left(\theta_{2}-1\right)^{2}-d_{M}^{2}=T\left(\theta_{1}, \theta_{2}\right)$, the condition for the Lagrangean maximum, defined as $U_{\theta_{1}} / U_{\theta_{2}}=T_{\theta_{1}} / T_{\theta_{2}}$, holds. Then, the maximum utility portfolio is given by $d_{M}$ and $r_{0}$ as shown in Figure 2.

\section{Comparing linear and quadratic metrics}

The bounding theorem is characterized by $L$ and $L_{\infty}$ in the normalized E-S space. Then, the vector $\boldsymbol{e}_{\boldsymbol{L}}$ starting at $L_{\infty}$ and ending at $L$ characterizes the bounding theorem. By translating the origin of coordinates from $(0,0)$ to point $L_{\infty}=\left(\theta_{1 \infty}, \theta_{2 \infty}\right)$, this vector $\boldsymbol{e}_{\boldsymbol{L}}$ presents the following properties:

1. A negative first coordinate of $\boldsymbol{e}_{\boldsymbol{L}}$ denotes a conservative bias or $r_{0}<1$. 


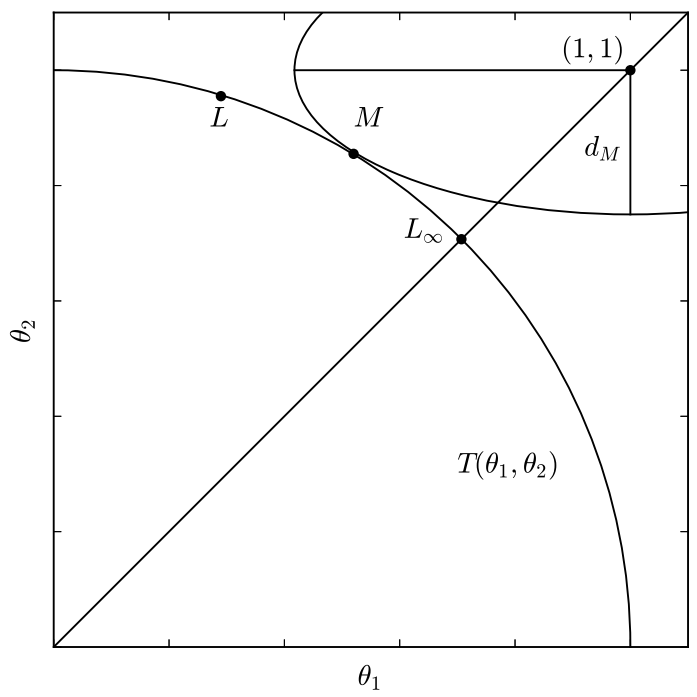

Fig. 2 Normalized efficient frontier $T\left(\theta_{1}, \theta_{2}\right)$ and position of relevant points in the characterization for Euclidean distances

2. A positive first coordinate of $\boldsymbol{e}_{\boldsymbol{L}}$ denotes a risky bias or $r_{0}>1$.

3. The norm $\left\|\boldsymbol{e}_{\boldsymbol{L}}\right\|$ is proportional to $r_{0}$.

4. The angle $\alpha$ of $\boldsymbol{e}_{\boldsymbol{L}}$ with respect to the x-axis is determined by $L$.

This vector $\boldsymbol{e}_{\boldsymbol{L}}$ does not only depend on an investor's particular risk preferences, but also on the efficient frontier and its particular form in the E-S space. Next, we use this vector $\boldsymbol{e}_{\boldsymbol{L}}$ when comparing balance of solutions.

Choosing the appropriate metric $\mathcal{L}_{h}$ in equation (5) is an ongoing issue in the compromise programming setting (Ballestero, 2007). Manhattan distances tend to outcome imbalanced solutions, i.e., close to the corner solutions $(1,0)$ and $(0,1)$ given by the intersection of $T\left(\theta_{1}, \theta_{2}\right)$ with the $\mathrm{x}$-axis and the $\mathrm{y}$-axis. On the other hand, Euclidean distances produce more balanced solutions.

Definition 1 We say that a solution $R=\left(\theta_{1 R}, \theta_{2 R}\right)$ is more balanced than $P=\left(\theta_{1 P}, \theta_{2 P}\right)$ if $R$ is closer than $P$ to $L_{\infty}$, in terms of Euclidean distance, i.e., if $\left\|\boldsymbol{e}_{\boldsymbol{R}}\right\|<\left\|\boldsymbol{e}_{\boldsymbol{P}}\right\|$, being $\boldsymbol{e}_{\boldsymbol{R}}$ and $\boldsymbol{e}_{\boldsymbol{P}}$ vectors from $L_{\infty}$ to $R$ and $L_{\infty}$ to $P$, respectively.

Within the limits set by Ballestero's bounding theorem, we next provide a necessary condition to obtain balanced solutions using metrics $\mathcal{L}_{1}$ and $\mathcal{L}_{2}$.

Theorem 4 Given an efficient frontier $T\left(\theta_{1}, \theta_{2}\right)$, a point $R$ within $L$ and $L_{\infty}$ maximizing utility $U_{2}$ is more balanced than point $P=\left(\theta_{1 P}, \theta_{2 P}\right)$ derived by maximizing $U_{1}$, when $\theta_{1}>d_{M} / \sqrt{2} r_{0}$. 
Proof From Theorem 2, we know that the slope of the tangent determining $P$ is $r_{0}$. Then, if $S$ is a more balanced solution than $P$, then the absolute value of the slope of the tangent line to $T\left(\theta_{1}, \theta_{2}\right)$ at $R$ must be greater than $r_{0}$. The slope of the tangent line to $T\left(\theta_{1}, \theta_{2}\right)$ at $R$ is given by the first derivative with respect to $\theta_{1}$ of the iso-ellipse equation:

$$
\theta_{2}^{\prime}=\frac{d \theta_{2}}{d \theta_{1}}=\frac{-r_{0}^{2} \theta_{1}}{\left[d_{M}^{2}-r_{0}^{2} \theta_{1}^{2}\right]^{1 / 2}}
$$

with $0<d_{M}<1$. If $\left|\theta_{2}^{\prime}\right|>r_{0}$ then:

$$
r_{0} \theta_{1}>\left[d_{M}^{2}-r_{0}^{2} \theta_{1}^{2}\right]^{1 / 2}
$$

which holds when:

$$
\theta_{1}>\frac{d_{M}}{\sqrt{2} r_{0}}
$$

\section{The balance theorem}

From the previous section, we know that solutions maximizing $U_{2}$ are more balanced than solutions maximizing $U_{1}$ when $\theta_{1}>d_{M} / \sqrt{2} r_{0}$ holds. This property can be generalized to $U_{h}$ through the following theorem:

Theorem 5 The Lagrangean maximum $M$ of the investors utility for metric $\mathcal{L}_{h}$ :

$$
U_{h}=K-\mathcal{L}_{h}=K-\left[r_{0}^{h}\left(1-\theta_{1}\right)^{h}+\left(1-\theta_{2}\right)^{h}\right]^{1 / h}
$$

between $L$ and $L_{\infty}$ for investors with risk preferences determined by $r_{0}>0$ is more balanced that the maximum for metric $\mathcal{L}_{h-1}$.

Proof From A8, we know that the condition for a minimum distance to the optimum is $U_{\theta_{1}} / U_{\theta_{2}}=T_{\theta_{1}} / T_{\theta_{2}}$, which is given by:

$$
\frac{d U_{h} / d \theta_{1}}{d U_{h} / d \theta_{2}}=\frac{r_{0} r_{0}^{h-1} \theta_{1}^{h-1}}{\theta_{2}^{h-1}}=\frac{T_{\theta_{1}}}{T_{\theta_{2}}} \quad \text { or } \quad \frac{\theta_{2}}{\theta_{1}}=\left[\frac{r_{0} T_{\theta_{2}}}{T_{\theta_{1}}}\right]^{\frac{1}{h-1}}
$$

which tends to one if $h$ tends to $\infty$ with $r_{0}>0$, and which is an increasing function of $h$ when $r_{0} T_{\theta_{2}} / T_{\theta_{1}}<0$, and a decreasing function of $h$ when $r_{0} T_{\theta_{2}} / T_{\theta_{1}}>0$. Then, as long as $h$ increases, the maximum moves closer to $L_{\infty}$, producing more balanced solutions according to Definition 2 .

From that, balance of solution $R$ defined by vector $\left\|\boldsymbol{e}_{\boldsymbol{R}}\left(\mathcal{L}_{h}\right)\right\|$ depending on metric $\mathcal{L}_{h}$ is proportional to $h$, i.e., vector norms are monotonically increasing with $h$ or $\left\|\boldsymbol{e}\left(\mathcal{L}_{h-1}\right)\right\| \leq\left\|\boldsymbol{e}\left(\mathcal{L}_{h}\right)\right\| \leq\left\|\boldsymbol{e}\left(\mathcal{L}_{h+1}\right)\right\|$.

Corollary 1 The most balanced solutions are produced using the Chebyshev distance $\mathcal{L}_{\infty}$. 


\section{Dealing with discrete efficient frontiers and uncertain risk preferences}

In practice, most of the efficient frontiers are discrete and most of the risk preferences are uncertain. Discrete efficient frontiers are the set of E-S points representing each of the available portfolios. On the other hand, risk preferences are uncertain in the sense that they are likely to be expressed in a loosely manner rather than as a single value $r_{0}$. For instance, my $r_{0}$ is around 1.5, neither higher than 2 nor lower than 1 . Here, we translate these expressions into a interval that is used as the main input to Algorithm 1 that selects the best set of compromise solutions. This algorithm outputs the best set of compromise solutions when given: (i) a discrete efficient frontier as a set of $n$ points, namely, $F=\left\{\left(\theta_{11}, \theta_{21}\right), \ldots,\left(\theta_{1 n}, \theta_{2 n}\right)\right\}$; (ii) an interval $\left[r_{\min }, r_{\max }\right]$; (iii) a metric $\mathcal{L}_{h}$.

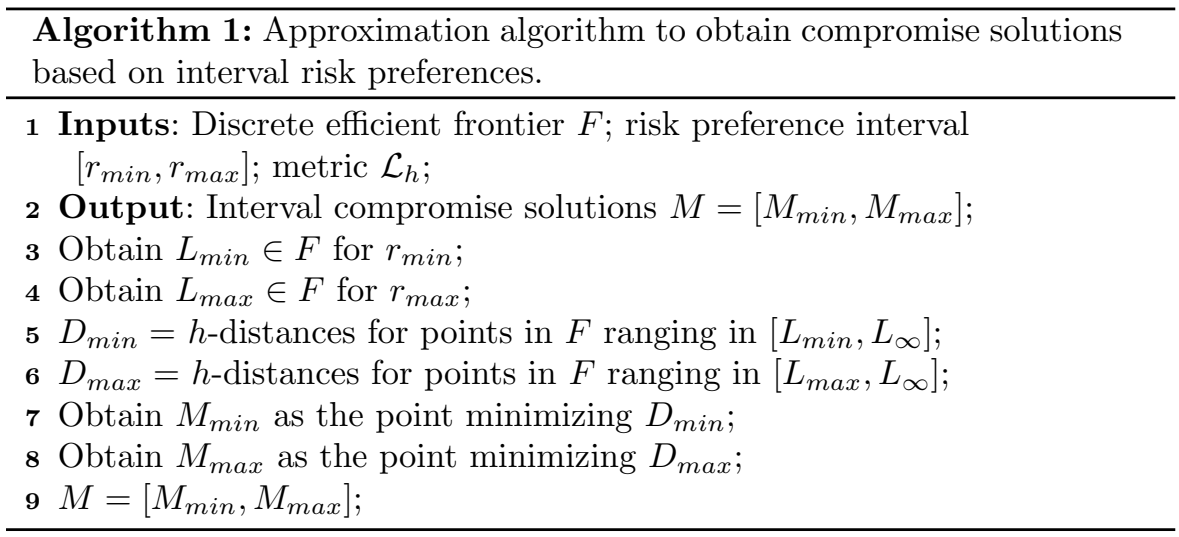

For illustrative purposes, let us consider the efficient frontier from the example given in Ballestero (1998) summarized in Table 1. Assume now that three different investors express their risk preferences using common words. Chances are that risk preferences are expressed as: (i) a declaration of being risky or conservative (recall that the efficient frontier has a risky zone when $\theta_{1}>\theta_{2}$, and a conservative zone when $\theta_{1}<\theta_{2}$ ); (ii) a threshold value, i.e., greater/less than a particular value; or (iii) as an interval, between a minimum and a maximum value. These expressions have an equivalent representation in the form of an interval $\left[r_{\min }, r_{\max }\right]$, that can be used as an input to Algorithm 1 to obtain the set of compromise solutions that fits their risk preferences. For instance, consider that the following hypothetical investors are willing to minimize distances to the ideal with metric $\mathcal{L}_{1}$ by means of Algorithm 1:

1. Investor 1: I am a conservative investor but I am unable to specify any particular risk preference, equivalent to $\left[r_{\min }, r_{\max }\right]=[0,1]$, since the conservative zone ranges in $0 \leq r_{0} \leq 1$. 
2. Investor 2: I am a risky investor but I am willing to loose no more than 1.5 units of safety to achieve an increase of one unit of profitability, equivalent to $\left[r_{\min }, r_{\max }\right]=[1,1.5]$

3. Investor 3: I am a risky investor but I am willing to loose neither more than 2 nor less than 1.5 units of safety to achieve an increase of one unit of profitability, equivalent to $\left[r_{\min }, r_{\max }\right]=[1.5,2]$.

Following the steps detailed in Algorithm 1, we can determine the set of portfolios that would satisfy each investor. As an example, consider Investor 1 with $\left[r_{\min }, r_{\max }\right]=[0,1]$. From Theorem 1 , we know that $L_{\min }=P_{1}$ and $L_{\max }=P_{5}$. In this case, $L_{\infty}$ is a point between $P_{5}$ and $P_{6}$, set to $(0.62,0.62)$ in the example by Ballestero (1998). Next, we compute Manhattan distances for each point in the efficient frontier ranging in $\left[P_{1}, L_{\infty}\right]$ and $\left[P_{5}, L_{\infty}\right]$, as shown in Table 1 . The rest of points are excluded from the analysis because the optimum does not lie among them according to Theorem 1. From this set of distances, we compute the minimum values to the ideal to determine the range that satisfies Investor1, namely, any portfolio between $P_{1}$ and $P_{5}$. Following the same procedure for the rest of investors, we obtain that any portfolio between $P_{5}$ to $P_{7}$ satisfies Investor 1 , and that any portfolio between $P_{7}$ to $P_{8}$ satisfies Investor 2 as detailed in Table 1 .

Table 1 Efficient frontier and satisfactory portfolios (marked with $\checkmark$ ) for different investors.

\begin{tabular}{crrrcccc} 
Portfolio & $\theta_{1}$ & $\theta_{2}$ & $\mathcal{L}_{1}\left(r_{0}=0\right)$ & $\mathcal{L}_{1}\left(r_{0}=1\right)$ & Investor 1 & Investor 2 & Investor 3 \\
\hline$P_{1}$ & 0 & 1 & 1.00 & - & $\checkmark$ & - & - \\
$P_{2}$ & 0.105 & 0.991 & 1.01 & - & $\checkmark$ & - & - \\
$P_{3}$ & 0.267 & 0.939 & 1.06 & - & $\checkmark$ & - & - \\
$P_{4}$ & 0.406 & 0.851 & 1.15 & - & $\checkmark$ & - & - \\
$P_{5}$ & 0.532 & 0.736 & 1.26 & 0.73 & $\checkmark$ & - \\
$P_{6}$ & 0.669 & 0.576 & - & - & - & - \\
$P_{7}$ & 0.803 & 0.389 & - & - & - & - \\
$P_{8}$ & 0.893 & 0.232 & - & - & - & - \\
$P_{9}$ & 1 & 0 & - & - & - & - & - \\
\hline
\end{tabular}

Further insight can be achieved through a graphical representation of the change of the best compromise solution for different values of the risk preference parameter $r_{0}$. Two consecutive points in any discrete efficient frontier present different values for $\theta_{1}$ and $\theta_{2}$. Then, plotting the variation of the particular values $\theta_{1}$ or $\theta_{2}$ of the best solution for $r_{0}$ ranging in $[0, \infty]$ shows how the best solution $M$ changes with $r_{0}$. Since $\theta_{2}$ is usually used as the vertical axis variable, here we choose $\theta_{2}$ to show changes in the best compromise solution. The outcome is a kind of decreasing stairs plot as shown in Figure 3. Just as the efficient frontier representation in Figures 1 and 2 is divided in two areas by the diagonal $\theta_{1}=\theta_{2}$, the stairs plot is divided by a vertical line $r_{0}=1$, delimiting the risky zone and the conservative zone.

The utility of such a plot is double: (i) risky/conservative investors should look for portfolios in the risky/conservative zone according to the their risk 


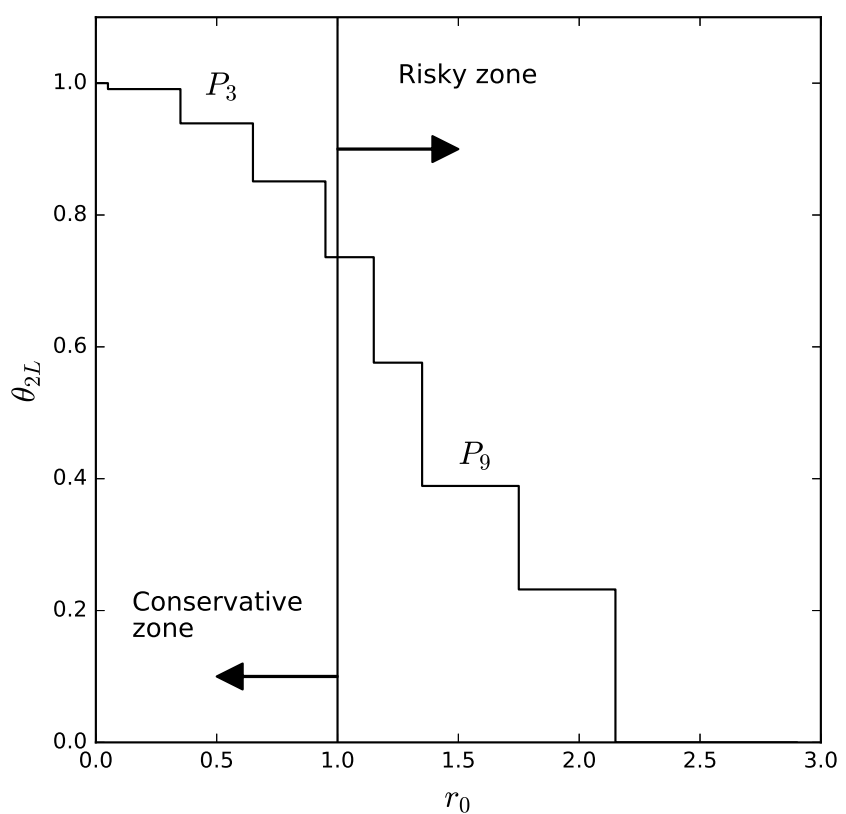

Fig. 3 Stairs plot: safety index $\theta_{2 M}$ of the best compromise solution $M$ for different risk preferences $r_{0}$ and metric $\mathcal{L}_{1}$

preferences, regardless of whether these preferences are defined by a single value or by an interval statement; (ii) validity of particular portfolios for different ranges of $r_{0}$ is immediately assessed by the width of the step of each portfolio, e.g., in Figure 3, portfolio $P_{7}$ is valid for a wider range of risk preferences than $P_{6}$, thus, $P_{7}$ can be considered a more stable portfolio.

Moreover, from the particular characteristics of the stairs plot, an interesting property of discrete efficient frontiers in contrast to continuous frontiers can be derived as follows:

Theorem 6 Given a discrete efficient frontier $F=\left\{\left(\theta_{11}, \theta_{21}\right), \ldots,\left(\theta_{1 n}, \theta_{2 n}\right)\right\}$, of $n$ points and a metric $\mathcal{L}_{h}$ with finite $h$, there exists a value $r_{\text {max }}$, which depends on $h$, such that the best compromise solution $M$ in a normalized space is always $(1,0)$ for all $r_{0}>r_{\max }$.

Proof For $h=1$, let $P=\left(\theta_{1 P}, \theta_{2 P}\right)$ be the next-to-last point and $(1,0)$ the last point in $F$, if $r_{\max }=\theta_{2 P} /\left(1-\theta_{1 P}\right)$, is the slope of the line passing through $P$ and $(1,0)$, all isolines with slope greater than $r_{\max }$ in absolute value are necessarily tangent to $F$ only in $(1,0)$. A similar reasoning leads to the same result for $h>1$. 
For instance, in the example from Table $1, r_{\max }=0.232 /(1-0.893)=2.2$, as reflected in the stairs plot of Figure 3. Recall that we are dealing with discrete efficient frontiers. In the case of continuous efficient frontiers, $r_{\max }=$ $\infty$ since the only isoline tangent to $(1,0)$ is a vertical line with infinite slope. Summarizing, it is important to highlight that: (i) risk preference intervals allow a wider and more flexible characterization than a single value; (ii) even though risk preferences are defined by an interval for a particular investor, the best compromise set of portfolios can be formed by a single portfolio; and (iii) a further step can be taken by considering not only interval but fuzzy risk preferences in which there is no sharp boundary between interval limits.

\section{Predicting long-run investor performance}

The basic idea in portfolio selection is diversification over a given set of available assets to achieve a maximum return for a certain level of risk. This idea assumes the existence of only one efficient frontier for a given set of available assets. However, chances are that different sets of assets produce different efficient frontiers and, eventually, different long-run performance. For instance, investors may be interested in selecting their best portfolio from assets located either in America, Europe or Asia. Other investors may focus on selecting assets either in the banking, industrial or telecommunications sector. Even within the same sector, investors may establish criteria to select companies according to their size. Determining the location, sector or company size that is expected to perform better and quantitatively estimating such improvement are two interesting challenges that are worth tackling. To this end, we propose the use of a single quality measure, namely, the Area Under the Curve (AUC).

Recall that the closer the portfolio to the ideal point the better the portfolio. Similarly, the closer the whole efficient frontier to the ideal point the better the efficient frontier. From that, a measure of quality of a particular efficient frontier in the normalized E-S space is the AUC, a widely used metric in machine learning (Fawcett, 2006; Hernández-Orallo et al., 2013) for assessing performance. In the context of portfolio selection, we define the AUC for a given efficient frontier $T\left(\theta_{1}, \theta_{2}\right)$ as:

$$
A U C\left(T, \theta_{1}, \theta_{2}\right)=\int_{0}^{1} T\left(\theta_{1}, \theta_{2}\right) d \theta_{1}
$$

ranging in $[0,1]$ and determining the average quality of portfolios obtained from a given set of available assets. For example, say that a group of investors consider alternative portfolios from assets located in Europe, America and Asia. After normalization, they derive three different efficient frontiers: $T_{1}$ for Europe; $T_{2}$ for America; and $T_{3}$ for Asia, as depicted in Figure 4. Clearly, portfolios in $T_{1}$ are better than those in $T_{2}$ (and portfolios in $T_{2}$ are better than those in $\left.T_{3}\right)$ since they are closer to the ideal point $(1,1)$. A measure that is able to quantitatively characterize which efficient frontier (location) is better (and how much better) in the long-term is the AUC. The group of investors in 
the example should invest in Europe since the expected performance should be better due to $\operatorname{AUC}\left(T_{1}, \theta_{1}, \theta_{2}\right)>A U C\left(T_{2}, \theta_{1}, \theta_{2}\right)>A U C\left(T_{3}, \theta_{1}, \theta_{2}\right)$. Generalizing, investors considering alternative sets of assets should first derive the efficient frontier for each set, then compute the AUC for each set, and finally select the set of assets with the higher AUC since the expected long-term performance is higher.

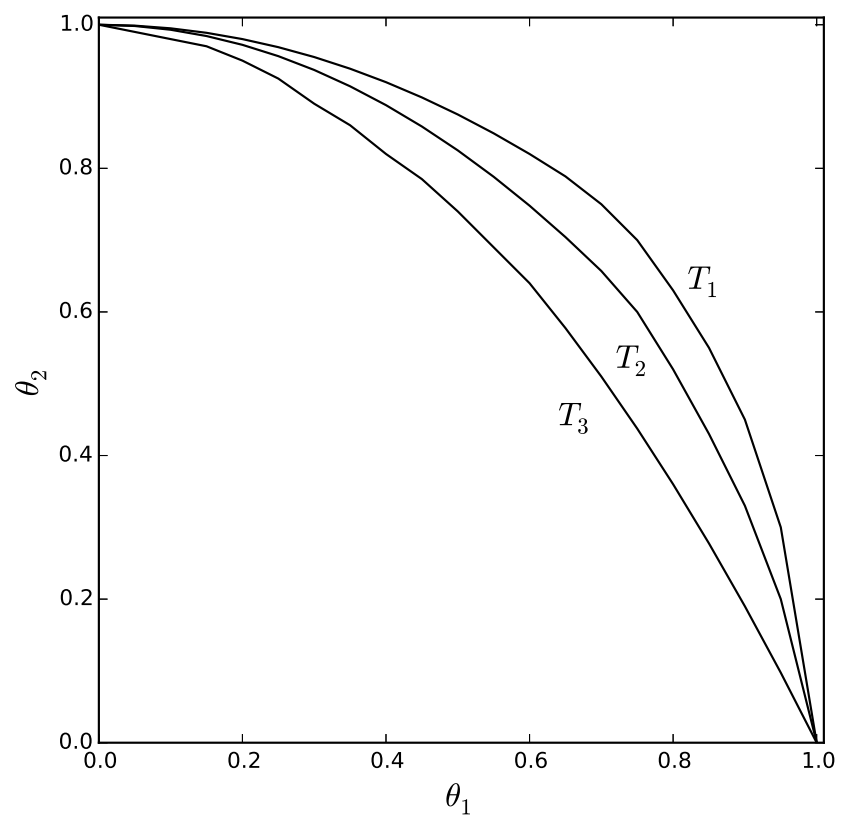

Fig. 4 Different efficient frontiers lead to different long-term performance.

However, when particular risk preferences of investors are considered, we know from Theorem 1 that the set of compromise solutions is bounded by $L$ and $L_{\infty}$. Then, the rest of available solutions are irrelevant to investors and can be discarded. From that, we propose a more refined measure of the quality for investors with particular risk preferences, which is the Area Between Bounds $(\mathrm{ABB})$, defined as:

$$
A B B\left(T, \tilde{\theta_{1}}, \tilde{\theta_{2}}\right)=\int_{0}^{1} T\left(\tilde{\theta_{1}}, \tilde{\theta_{2}}\right) d \tilde{\theta_{1}}
$$

where $T\left(\tilde{\theta_{1}}, \tilde{\theta_{2}}\right)$ is a re-scaled efficient frontier when the point $L$ is now the corner solution $(0,1)$ and $L_{\infty}$ is the other corner solution $(1,0)$. The procedure is shown in Figure 5. After deriving $L$ from $r_{0}$, a new origin of coordinates is 
determined by the intersection of the vertical line containing $L$ and the horizontal line containing $L_{\infty}$. For meaningful comparisons, the efficient frontier is re-scaled to be fitted in a new normalized E-S space by mapping $L$ to $(0,1)$ and $L_{\infty}$ to $(1,0)$.

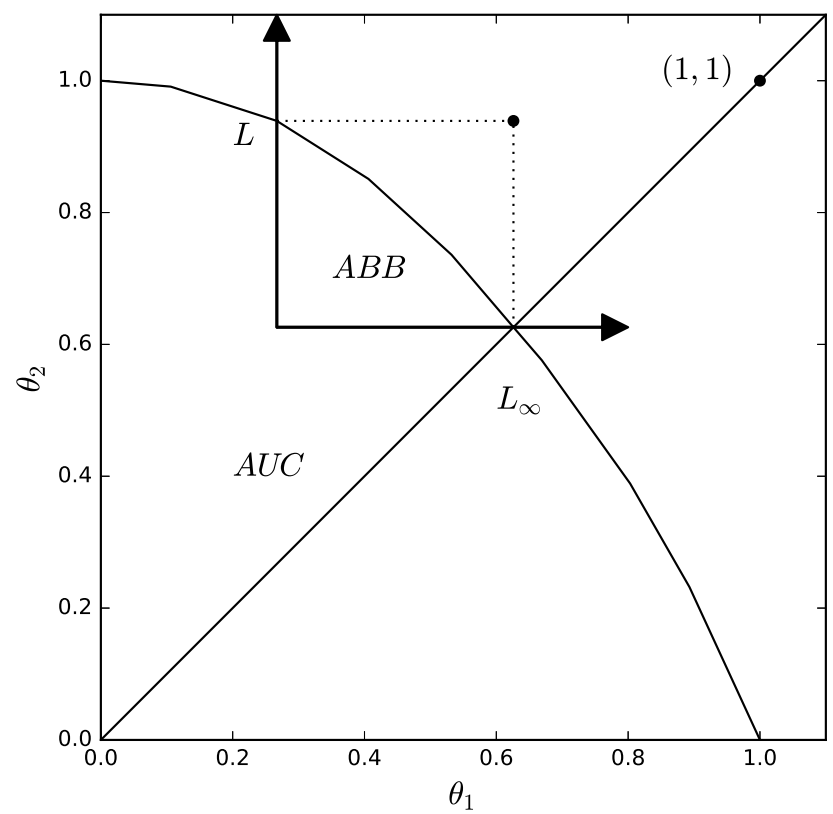

Fig. 5 Two different measures to predict long-run performance: the Area Under the Curve (AUC) and the Area Between Bounds (ABB)

The utility of the $A B B$ metric is better understood through the following example. Assume that two different investors, $I_{1}$ and $I_{2}$, want to select portfolios from the same set of available assets that produces $T\left(\theta_{1}, \theta_{2}\right)$ with $A U C=0.7$. Assume also that $I_{1}$ is a risky investor with risk preference given by $r_{1}=1.5$ and that $I_{2}$ is a conservative investor with risk preference given by $r_{2}=0.5$. After deriving $L$ for both $r_{1}$ and $r_{2}$, re-scaling $T$ and computing $A B B$ for both investors, assume that the results yield $A B B_{1}=0.6$ and $A B B_{2}=0.55$ respectively. From that, we infer that investor $I_{1}$ would outperform $I_{2}$ since $A B B_{1}>A B B_{2}$. This different performance denotes a certain bias of $T$ that would act in favor of risky investors and that can be measured by the proposed metric $A B B$.

Summarizing, the $A U C$ metric help us predict the long-run performance of investors selecting portfolios from different sets of available assets. On the other hand, the $A B B$ metric help us predict the long-run performance of investors 
with particular risk preferences since portfolios located out of the limits $L$ and $L_{\infty}$ are irrelevant to them.

\section{Conclusions}

In this paper, we characterize Ballestero's bounding theorem in portfolio selection for investors with particular risk preferences. We claim this characterization is necessary since investors are more interested in finding a particular optimal solution rather than a set between two bounds. Visual techniques allow to enhance the understanding of decision-making techniques based on compromise programming. Even though this paper particularizes on two specific loss functions, namely, $\mathcal{L}_{1}$ for Theorem 2 and $\mathcal{L}_{2}$ for Theorem 3 , it is likely that no other loss function is used in practice due to computational and interpretation problems. Further insight in the characterization of the bounded compromise set is given. More precisely, we show the utility of:

1. Theorem 4 determining the necessary condition to obtain more balanced solutions using the linear and quadratic common metrics in compromise programming. This condition depends on the particular risk preferences of investors.

2. Theorem 5 showing the balance relation of solutions obtained using different loss functions $\mathcal{L}_{h}$. This theorem implies that the balance of solutions depending on metric $\mathcal{L}_{h}$ monotonically increases with $h$.

3. An algorithm and a stairs plot to show the variation of solutions for discrete efficient frontiers and risk preferences expressed as a single value $r_{0}$ or as an interval statement.

4. Two quality measures to predict long-run investor performance, the Area Under the Curve and the Area Between Bounds which is directly linked to Ballestero's bounding theorem.

Finally, it is important to point out that visual techniques help enhance the understanding of complex decision-making problems such as portfolio optimization. Investors with particular risk preferences can rely on this kind of techniques to make better decisions. Plots and metrics allow a more informed decision-making process. Discrete efficient frontiers and risk preference intervals for particular criteria introduces a new practical perspective to multiobjective decision problems that deserves further research.

\section{References}

Amiri, M., Ekhtiari, M., and Yazdani, M. (2011). Nadir compromise programming: A model for optimization of multi-objective portfolio problem. Expert Systems with Applications, 38(6):7222-7226.

Ballestero, E. (1998). Approximating the optimum portfolio for an investor with particular preferences. Journal of the Operational Research Society, pages $998-1000$. 
Ballestero, E. (2007). Compromise programming: A utility-based linearquadratic composite metric from the trade-off between achievement and balanced (non-corner) solutions. European journal of operational research, 182(3):1369-1382.

Ballestero, E. and Pla-Santamaria, D. (2004). Selecting portfolios for mutual funds. Omega, 32(5):385-394.

Ballestero, E., Pla-Santamaria, D., Garcia-Bernabeu, A., and Hilario, A. (2015). Portfolio selection by compromise programming. In Socially Responsible Investment, pages 177-196. Springer.

Ballestero, E. and Romero, C. (1996). Portfolio selection: A compromise programming solution. Journal of the Operational Research Society, 47(11):1377-1386.

Ballestero, E. and Romero, C. (1998). Multiple criteria decision making and its applications to economic problems. Kluwer Academic Publishers.

Bilbao-Terol, A., Pérez-Gladish, B., Arenas-Parra, M., and Rodríguez-Uría, M. V. (2006). Fuzzy compromise programming for portfolio selection. Applied Mathematics and computation, 173(1):251-264.

Bravo, M., Ballestero, E., and Pla-Santamaria, D. (2012). Evaluating fund performance by compromise programming with linear-quadratic composite metric: An actual case on the caixabank in spain. Journal of Multi-Criteria Decision Analysis, 19(5-6):247-255.

Ehrgott, M., Klamroth, K., and Schwehm, C. (2004). An mcdm approach to portfolio optimization. European Journal of Operational Research, 155(3):752-770.

Fawcett, T. (2006). An introduction to roc analysis. Pattern recognition letters, $27(8): 861-874$.

Hernández-Orallo, J., Flach, P., and Ferri, C. (2013). Roc curves in cost space. Machine learning, 93(1):71-91.

Markowitz, H. (1952). Portfolio selection. The journal of finance, 7(1):77-91.

Pla-Santamaria, D. and Bravo, M. (2013). Portfolio optimization based on downside risk: a mean-semivariance efficient frontier from dow jones blue chips. Annals of Operations Research, 205(1):189-201.

Ringuest, J. L. (1992). Multiobjective optimization: behavioral and computational considerations. Springer Science \& Business Media.

Steuer, R. E., Qi, Y., and Hirschberger, M. (2007). Suitable-portfolio investors, nondominated frontier sensitivity, and the effect of multiple objectives on standard portfolio selection. Annals of Operations Research, 152(1):297-317.

Xidonas, P., Mavrotas, G., Krintas, T., Psarras, J., and Zopounidis, C. (2012). Multicriteria portfolio management. Springer.

$\mathrm{Yu}$, P.-L. (1973). A class of solutions for group decision problems. Management Science, 19(8):936-946.

Yu, P.-L. (1985). Multiple criteria decision making: concepts, techniques and extensions. Plenum Press.

Zeleny, M. (1982). Multiple criteria decision making. McGraw-Hill. 